\title{
Transition to convection in single bubble diffusive growth
}

\author{
Álvaro Moreno Soto ${ }^{1, \dagger}$, Oscar R. Enríquez ${ }^{2} \dagger$, Andrea Prosperetti ${ }^{3}$, \\ Detlef Lohse ${ }^{1}$ and Devaraj van der Meer \\ ${ }^{1}$ Physics of Fluids Group and Max Planck Center Twente, MESA+ Institute and J. M. Burgers Centre \\ for Fluid Dynamics, Faculty of Science and Technology, University of Twente, P.O. Box 217, \\ 7500 AE Enschede, The Netherlands \\ ${ }^{2}$ Fluid Mechanics Group, Universidad Carlos III de Madrid, Avda. de la Universidad 30, \\ 28911 Leganés (Madrid), Spain \\ ${ }^{3}$ Department of Mechanical Engineering, University of Houston, 4726 Calhoun Rd, Houston, USA
}

(Received 12 November 2018; revised 29 March 2019; accepted 2 April 2019;

first published online 20 May 2019)

We investigate the growth of gas bubbles in a water solution at rest with a supersaturation level that is generally associated with diffusive mass transfer. For $\mathrm{CO}_{2}$ bubbles, it has been previously observed that, after some time of growing in a diffusive regime, a density-driven convective flow enhances the mass transfer rate into the bubble. This is due to the lower density of the gas-depleted liquid which surrounds the bubble. In this work, we report on experiments with different supersaturation values, measuring the time $t_{\text {conv }}$ it takes for convection to dominate over the diffusion-driven growth. We demonstrate that by considering buoyancy and drag forces on the depleted liquid around the bubble, we can satisfactorily predict the transition time. In fact, our analysis shows that this onset does not only depend on the supersaturation, but also on the absolute pressure, which we corroborate in experiments. Subsequently, we study how the depletion caused by the growth of successive single bubbles influences the onset of convection. Finally, we study the convection onset around diffusively growing nitrogen $\mathrm{N}_{2}$ bubbles. As $\mathrm{N}_{2}$ is much less soluble in water, the growth takes much longer. However, after waiting long enough and consistent with our theory, convection still occurs as for any gas-liquid combination, provided that the density of the solution sufficiently changes with the gas concentration.

Key words: bubble dynamics, buoyant boundary layers

\section{Introduction}

The physics of bubble growth in mildly supersaturated solutions is potentially relevant to several processes associated with energy production and consumption, e.g. increased production rate in oil generation (Pooladi-Darvish \& Firoozabadi

$\dagger$ †mail addresses for correspondence: a.morenosoto@utwente.nl, oenrique@ing.uc3m.es 
1999; Akin \& Kovscek 2002). Alternative energy generation methods such as syngas (Munasinghe \& Khanal 2010), photo-electrochemical hydrolysis to obtain hydrogen (Leenheer \& Atwater 2010; Spurgeon \& Lewis 2011; Fernández et al. 2014) and catalytic reactions (Somorjai \& Li 2010) must deal with multi-phase systems which are often affected by bubble formation. As one last example, $\mathrm{CO}_{2}$ bubble formation is an undesired event in carbon sequestration, where $\mathrm{CO}_{2}$ is injected at high pressures into reservoirs of saline water trapped in porous rocks at depth (Neufeld et al. 2010; Tsai, Riesing \& Stone 2013; Bolster 2014). It appears sensible to assume that in liquids at rest such slow bubble growth (or dissolution) takes place exclusively by diffusion, as in the seminal theoretical analysis by Epstein \& Plesset (1950), which predicts that the bubble radius $R$ evolves in a pure diffusive regime as

$$
R(t) \approx\left(\sqrt{\frac{c_{0}-c_{s}}{2 \pi \rho_{g}}}+\sqrt{1+\frac{c_{0}-c_{s}}{2 \pi \rho_{g}}}\right) \sqrt{\frac{2 D\left(c_{0}-c_{s}\right)}{\rho_{g}} t} t .
$$

Here, $c_{0}$ is the concentration in the bulk liquid at the initial saturation pressure $P_{0}$, $c_{s}$ is the supersaturated concentration at the ambient pressure $P_{s}$ during the experiments, $\rho_{g}$ is the gas density and $D$ is the diffusion coefficient of the dissolved gas in the liquid. Such time evolution of the bubble radius has been confirmed in several experimental works, with supersaturations $\zeta=\left(c_{0}-c_{s}\right) / c_{s}$ comparable to that of carbonated beverages $(\zeta \sim 1-3)$ (Bisperink \& Prins 1994; Jones, Evans \& Galvin 1999b; Barker, Jefferson \& Judd 2002; Li et al. 2014).

However, in previous experimental studies of single $\mathrm{CO}_{2}$ bubbles growing on a silicon substrate in very mildly supersaturated solutions with $\zeta \sim 0.1-0.3$ (Enríquez et al. 2014; Moreno Soto et al. 2017), significant differences were observed with respect to a purely diffusive growth. The most notable discrepancy consists of an enhanced mass transfer rate towards the later stages of the bubble growth, which exceeds the predicted diffusive growth rate and corresponds to a time evolution of the bubble radius different from $R \propto \sqrt{t}$. This behaviour originates from the development of buoyancy-driven convection induced by the decreased density of the $\mathrm{CO}_{2}$-depleted liquid around the bubble. It has been shown that buoyancy-driven convection is also the cause of higher dissolution rates of sessile droplets in a less dense liquid (Dietrich et al. 2016) and during droplet evaporation (Shahidzadeh-Bonn et al. 2006). Both situations are physically analogous to growing bubbles and customarily treated as purely diffusion-driven phenomena (Popov 2005; Stauber et al. 2014; Lohse \& Zhang 2015), neglecting convective effects.

In this article, we further delve into the development of natural convection around a single growing bubble. We focus in particular on the time that it takes for convection to set in as an appreciable mass transfer mechanism and what external conditions, such as the concentration in the bulk liquid $c_{0}$ and the supersaturation level $\zeta$, have more influence on its transition. We present a simple theoretical criterion to predict the time $t_{c o n v}$ at which convection becomes important and find a good agreement with experimental measurements using $\mathrm{CO}_{2}$ bubbles. Afterwards, the long-term effects of depletion in a single bubble succession are investigated, showing a significant influence on $t_{c o n v}$ and the strength with which convection sets in. Finally, two different gases in solution $\left(\mathrm{CO}_{2}\right.$ and $\left.\mathrm{N}_{2}\right)$ are contrasted, revealing a unique behavioural change in the mass transfer rate at the predicted time $t_{c o n v}$ due to the different gas properties. 


\section{Experimental set-up and theoretical approach}

Our experiments start with an equilibrated solution of either $\mathrm{CO}_{2}$ or $\mathrm{N}_{2}$ in ultrapure degassed water at a starting saturation pressure $P_{0}$. The initial dissolved gas concentration $c_{0}$ is given by Henry's law, i.e. $c_{0}=k_{H} P_{0}$, where $k_{H}$ depends on the gas-liquid couple and the temperature $T$. We then drop the pressure isothermally $(T \approx$ $20^{\circ} \mathrm{C}$ ) to $P_{s}$ in order to induce a supersaturation

$$
\zeta=\frac{c_{0}-c_{s}}{c_{s}}=\frac{P_{0}-P_{s}}{P_{s}} .
$$

The pressure controller induces some oscillations after the pressure drop, which can be slightly detected in the figures shown later. Due to this pressure decrease, a bubble grows out of a hydrophobised micro-pit of radius $R_{p}=10 \mu \mathrm{m}$ or $50 \mu \mathrm{m}$ which is covered with black silicon (Jansen et al. 1995) and acts as an artificial bubble nucleation site (Borkent et al. 2009). The size of the pit, etched on a silicon substrate, determines the minimum supersaturation for which a bubble grows (Jones, Evans \& Galvin 1999a; Enríquez 2015; van der Linde et al. 2018), $\zeta>2 \sigma / P_{s} R_{p}$, which typically corresponds to a value $\zeta \approx 0.07$. However, it is extremely difficult to achieve bubble formation for this value due to the limitations of our set-up. On the other limit, very high $c_{s}$ or $\zeta$ imply uncontrolled bubble nucleation in areas outside the field of view. This interferes with the proper analysis of the target bubble due to massive bubble formation. $R_{p}$ also defines the bubble detachment radius (known as the Fritz radius; Fritz (1935)) by equilibrating capillary and buoyancy forces:

$$
R_{\text {det }}=\left(\frac{3 R_{p} \sigma}{2\left(\rho_{m}-\rho_{g}\right) g}\right)^{1 / 3},
$$

where $\sigma$ is the surface tension coefficient, $\rho_{m}$ is the solution density and $g$ is the gravitational acceleration. The pit is located so that the bubble growing atop is far from the holding device, where several bubbles usually grow, and also far enough from the edges of the substrates, where parasitic bubbles might grow on the microroughness caused by the dicing process through which an original silicon wafer is cut into the shape of the experimental chips. Unless otherwise indicated, the bubbles always grow on top of the substrate and all experiments are done in the range $0.1<$ $\zeta<0.5$; higher supersaturations already result in too many bubbles on the walls of the tank and on the edges of the silicon substrate and, consequently, it can no longer be ensured that the liquid is at rest. Figure 1(a) shows a sketch of the experimental set-up. A detailed description can be found in Enríquez et al. (2013).

Figure $1(b)$ shows a sketch of a growing bubble. The Laplace pressure due to the surface tension is in our case very small compared to $P_{s}$ and can be safely neglected. Hence, the gas concentration at the interface can be considered constant and equal to $c_{s}$ and, therefore, the bubble grows due to the diffusive gas flow driven by the concentration difference $c_{s} \zeta$ and the gas diffusivity $D$. The idealised initial condition is that the bubble grows from a radius $R_{p}$ with concentration $c_{s}$ at the interface and $c_{0}$ everywhere else. The asymptotic solution for the growth of a bubble in an infinite medium with the aforementioned initial conditions (1.1) (Epstein \& Plesset 1950) needs to be adapted to account for the presence of the silicon substrate below the bubble interface (Enríquez et al. 2014), which obstructs the mass transfer towards the bubble. The corrected asymptotic solution thus reads:

$$
\epsilon \approx\left(\sqrt{\frac{c_{s} \zeta}{2 \pi \rho_{g}}}+\sqrt{\frac{1}{2}+\frac{c_{s} \zeta}{2 \pi \rho_{g}}}\right) x \equiv S^{*} x,
$$


(a)

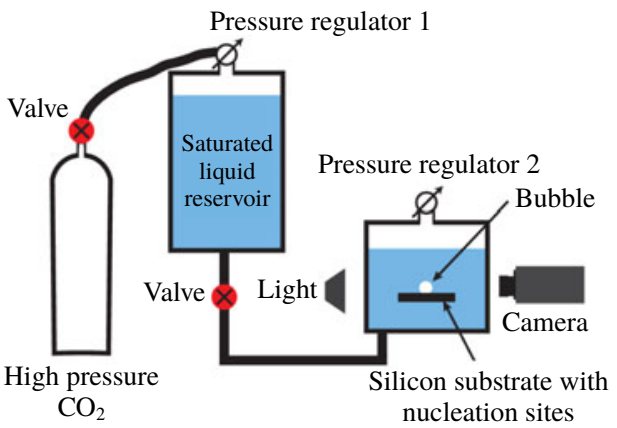

(b)

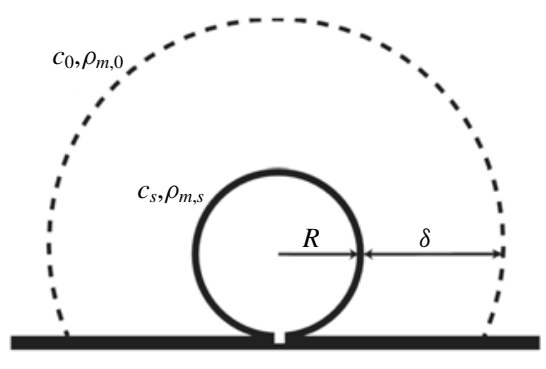

FIGURE 1. (Colour online) Sketches of $(a)$ the experimental set-up and $(b)$ the growing bubble. (a) A saturated water- $\mathrm{CO}_{2}$ solution is prepared in the reservoir tank at a pressure $P_{0}$. After transferring part of the mix to the observation tank, the pressure is dropped to $P_{s}$ in order to produce a small supersaturation. A bubble grows from a nucleation site defined by a single hydrophobic cavity etched in a silicon substrate. We record the process using a long distance microscope objective through a lateral window of the tank. (b) As the bubble radius $R$ grows, it develops a concentration $c$ and density $\rho_{m}$ profile of thickness $\delta(t)$.

where $\epsilon=R / R_{p}$ is the dimensionless bubble radius and $x=\sqrt{\left(2 D c_{s} \zeta t\right) /\left(\rho_{g} R_{p}^{2}\right)}$. Note that $R_{p}$ works in (2.3) as a reference value and does not directly affect the growth rate (Barker et al. 2002). $S^{*}$ typically reaches values around 0.9 and it increases with higher $\zeta$ and $P_{0}$. To compare experiments with this theoretical behaviour, a time origin needs to be determined. We measure it in two steps: firstly, we identify $t_{0}=0$ with the moment in which we first detect a bubble protruding from the pit; secondly, we numerically fit a function $\sqrt{t}$ (i.e. the theoretical behaviour for pure diffusion) to the early stages of the experimental bubble growth (ignoring the first few data points associated with the growth of the bubble out of the pit). We correct the former $t_{0}$, which was already within the error limit $\pm 0.5 \mathrm{~s}$.

As the bubble grows, it depletes its surroundings of gas (Moreno Soto et al. 2017), developing a diffusively growing concentration profile that extends a distance $\delta=$ $\sqrt{\pi D t}$ into the liquid solution (figure $1 b$ ). Variations in concentration imply changes in the solution density, determined by the concentration expansion coefficient $\lambda_{c}$, which for a dilute solution of gas in water can be approximated as (Gebhart \& Pera 1971; Moreno Soto et al. 2017; Peñas-López et al. 2017):

$$
\lambda_{c}=\frac{1}{\rho}\left(\frac{\partial \rho}{\partial c}\right)_{P, T} \approx \frac{1}{\rho_{m, 0}} \frac{\rho_{m, 0}-\rho_{m, s}}{c_{0}-c_{s}} .
$$

Hence, a positive $\lambda_{c}$ means that the density of the solution increases with the gas concentration. If the density of the saturated solution is $\rho_{m, 0}$, the density value at the bubble surface is given by $\rho_{m, s}=\rho_{m, 0}\left(1-\lambda_{c} c_{s} \zeta\right)$. The different gas properties of $\mathrm{CO}_{2}$ and $\mathrm{N}_{2}$ are listed in table 1. Combining $\lambda_{c}$ with $c_{s} \zeta \approx 2 \mathrm{~kg} \mathrm{~m}^{-3}$ (for the $\mathrm{CO}_{2}$ experiments; $c_{s} \zeta$ is much smaller for the $\mathrm{N}_{2}$ case) implies that $\left|\lambda_{c} c_{s} \zeta\right| \ll 1$ and makes it tempting to neglect the changes in density, which has been routinely done in most earlier works on bubble growth in supersaturated solutions. However, the long growth times of the bubbles require that we do take long term density changes into consideration. 
$D\left(\mathrm{~m}^{2} \mathrm{~s}^{-1}\right)$ (Wilke \& Chang 1955)

$k_{H}\left(\mathrm{~kg}\left(\mathrm{~m}^{-3} \mathrm{~Pa}^{-1}\right)\right)($ Sander 2015)

$\lambda_{c}\left(\mathrm{~m}^{3} \mathrm{~kg}^{-1}\right)$ (Equation (2.4) and Watanabe \& Iizuka (1985))

$\rho_{g}\left(\mathrm{~kg} \mathrm{~m}^{-3}\right)$ (Greenwood \& Earnshaw 1997; Pierantozzi 2007)
$\mathrm{CO}_{2}$

$\mathrm{N}_{2}$

$\begin{array}{cc}1.79 \times 10^{-9} & 1.88 \times 10^{-9} \\ 1.67 \times 10^{-5} & 1.78 \times 10^{-7} \\ 9.90 \times 10^{-4} & -2.32 \times 10^{-4} \\ 9.97 & 6.29\end{array}$

TABLE 1. Properties of $\mathrm{CO}_{2}$ and $\mathrm{N}_{2}$ when dissolved in water at $P=0.55 \mathrm{MPa}$ and $T=21^{\circ} \mathrm{C}$. The large difference in the values of Henry's constant $k_{H}$ accounts for the two orders of magnitude change in solubility.

As will be thoroughly explained in the following section, after an initial diffusive growth, a transition to density-driven natural convection occurs. In order to experimentally measure the time in which this transition takes place, two methods are used and compared. An analytical model comparing buoyant and viscous forces will be defined to theoretically approximate this transition time, which only depends on the properties of the solution and the supersaturation level $\zeta$ at which bubbles grow. Whereas for a purely diffusively growing bubble (2.3) the ratio between the bubble radius and its diffusive concentration boundary layer $R / \delta=S^{*} \sqrt{2 c_{s} \zeta / \pi \rho_{g}}$ remains constant through time, the transition to convection enhances the bubble growth rate but does not influence $\delta$. Thus, the ratio $R / \delta$ increases as compared to a purely diffusive case. Higher supersaturation levels $\zeta$ and $P_{0}$ (which directly relates to $c_{s}$ ) also make this ratio increase. However, within this relatively smaller concentration layer, higher concentration gradients are reached, which results in higher and intensified onset to natural convection, as we will analyse in the following section.

\section{The case of $\mathrm{CO}_{2}$ bubbles}

The typical bubble growth from our experiments is best appreciated by plotting the derivative $\mathrm{d} \epsilon / \mathrm{d} x$, which represents the dimensionless rate of change of the bubble area. Following (2.3) this should be constant and approximately equal to $S^{*}$. Figure $2(a)$ shows $\mathrm{d} \epsilon / \mathrm{d} x$ divided by $S^{*}$ for some experiments with $\mathrm{CO}_{2}$ solutions at $P_{0}=0.35 \mathrm{MPa}$ and different $\zeta$. After the initial transient associated with the sudden growth of the bubble out of the pit, there is indeed a plateau around $\left(1 / S^{*}\right)(\mathrm{d} \epsilon / \mathrm{d} x) \approx 1$, which indicates a diffusion-driven growth. The plateau reaches larger values with increasing $\zeta$. This originates from the stronger bubble growth rate at larger supersaturation levels $\zeta$, which the asymptotic model (2.3) cannot follow any longer. Nevertheless, for the experimental range studied, the expected plateau corresponding to a diffusive process is always found. Afterwards, the curves start to rise as a result of the enhancement of mass transfer caused by natural convection. To properly associate this effect with a transition to natural convection, following Enríquez et al. (2014), we introduce the Sherwood number $S h$ and the mass Rayleigh number $R a$, respectively defined as:

$$
S h=\frac{2 R \dot{R} \rho_{g}}{D c_{s} \zeta}, \quad R a=\frac{g \lambda_{c} c_{s} \zeta(2 R)^{3}}{\nu D},
$$

where $\dot{R}$ is the (dimensional) bubble growth rate and $v$ is the kinematic viscosity of water. Defined this way, $S h$ stands for the dimensionless mass transfer rate towards the bubble and $R a$ for the dimensionless buoyancy force due to the concentration 

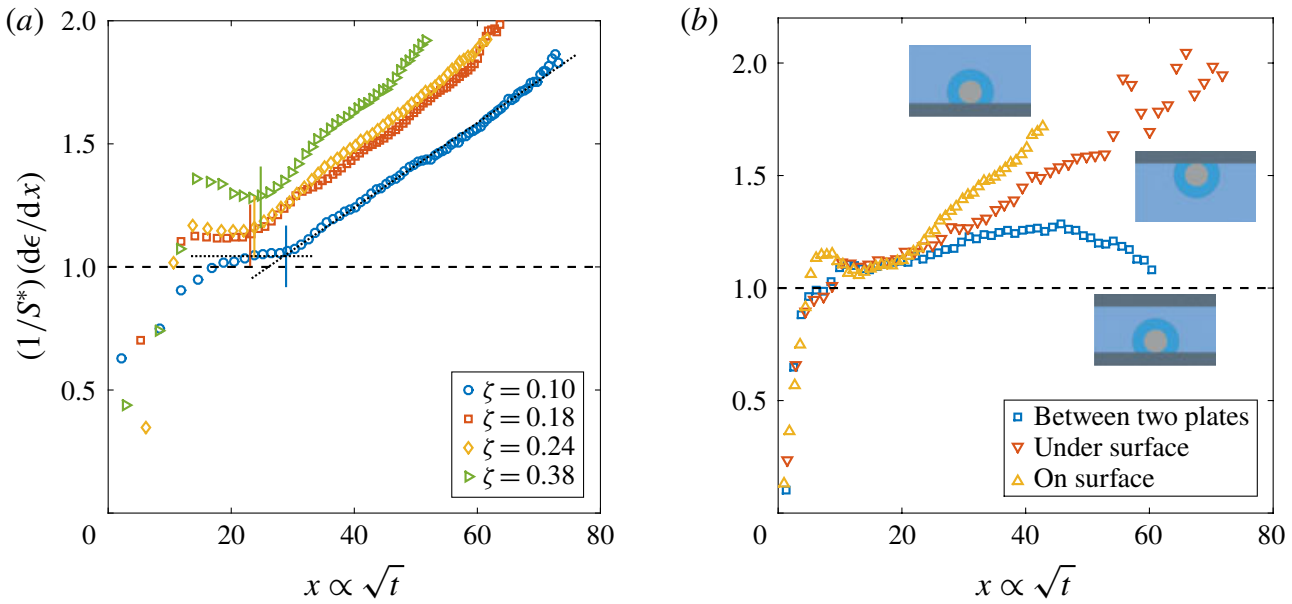

FiguRE 2. (Colour online) Dimensionless bubble growth rate (or, equivalently, the dimensionless change in the bubble surface over time) divided by $S^{*}$ as a function of $x \propto \sqrt{t}$. The leftmost rising part associated with the sudden growth of the bubble out of the pit and the following horizontal plateau are expected from a diffusion-driven growth; the right-hand slope suggests the influence of convection. The horizontal dashed line represents the purely diffusive evolution according to (2.3). (a) Experiments at $P_{0}=$ $0.35 \mathrm{MPa}$ and a pit of $R_{p}=50 \mu \mathrm{m}$ with various supersaturation levels $\zeta$. In all cases, the bubble grows on top of the silicon chip. The vertical solid lines in corresponding colours indicate the experimentally measured dimensionless square root of the transition time $x_{c}$ for each $\zeta$. The two reference lines used on its calculation are shown as dotted lines for $\zeta=0.10$. (b) Experiments at $P_{0}=0.65 \mathrm{MPa}$, a pit of $R_{p}=10 \mu \mathrm{m}$ and $\zeta \approx 0.2$ with the bubble growing in different geometrical configurations, indicated by the sketches next to the corresponding curves.

difference in the liquid. When $S h$ is plotted against $R a$ (figure $3 a$ ), two main phases can be distinguished. Firstly, an initial transient stabilises to a plateau value which reads $S h=2 S^{* 2}$ for pure diffusive growth. For higher supersaturation $\zeta$, this plateau is expected to reach slightly higher values, as $S^{*}$ increases accordingly with $\zeta$, equation (2.3). Secondly, the rising part of the curve follows a power law $S h \propto R a^{1 / 4}$, which is the expected relation between $S h$ and $R a$ for natural convection around a sphere (see e.g. Potter \& Riley 1980; Bejan 1993). Further experimental confirmation of the presence and influence of natural convection is obtained by comparing the bubble growth in different geometrical configurations, such as the situation where the bubble grows underneath the substrate and where it grows between two horizontal parallel plates with a vertical separation of $1 \mathrm{~mm}$ (a distance approximately 2.5 times larger than the bubble detachment radius), figure $2(b)$. In the first case, an increase of the growth rate is still observed, but at a slower pace, which is to be expected because of the geometrical inversion of the problem where the buoyant depleted liquid needs to move sideways due to the presence of the substrate. In the second case, the growth rate starts to rise but decreases again as the bubble surface approaches the other wall, which inhibits the possibility of convection developing further. We anticipate the different configurations to affect the Riley scaling law, more precisely on the prefactor multiplying $R a^{1 / 4}$. This power law has also been demonstrated for natural convection above and below a heated plate (Bejan 1993, chap. 7, § 7.3.3). 

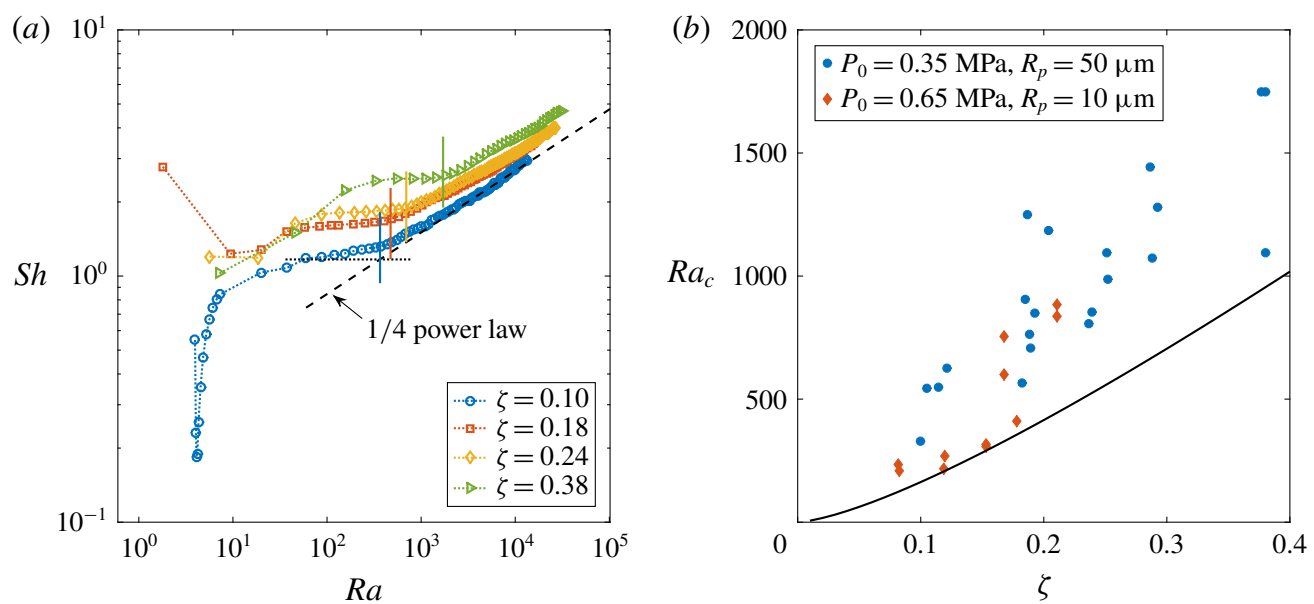

FIgURE 3. (Colour online) (a) Sherwood number $S h$ as a function of Rayleigh number $R a$ in double logarithmic scale from the same data presented in figure 2(a). The dashed line represents a $1 / 4$ power law, which indicates that in the advanced stages, growth is driven by natural convection (Enríquez et al. 2014; Moreno Soto et al. 2017). The dotted horizontal line stands for a purely diffusive growth and its intersection with the power law indicates the transition to convection. The vertical solid lines in corresponding colours indicate the measured $R a_{c}$ for each curve. (b) Rayleigh number $R a_{c}$ at the cross-over to convection-driven growth as a function of the supersaturation $\zeta$. Blue circles correspond to experiments with $P_{0}=0.35 \mathrm{MPa}$ and $R_{p}=50 \mu \mathrm{m}$ and red diamonds to $P_{0}=0.65 \mathrm{MPa}$ and $R_{p}=10 \mu \mathrm{m}$. The solid black line represents the theoretical $R a_{c}$ as given by (3.7), which does not depend on $P_{0}$ and therefore is coincidental for the two cases presented.

\subsection{Transition to convection-driven growth}

To characterise the cross-over to convection-driven growth in experiments, we fit a horizontal line to the plateau region of the $S h$ versus $R a$ curves (figure $3 a$ ) and a 1/4-power law to their rising part and assume that the transition takes place at the intersection of those two lines. The crossing $R a_{c}$ can be directly associated with a bubble radius $R$ and thus directly translated to a transition time $t_{c o n v}$. Equivalently, we also fit a horizontal line to the plateau in the derivative curves in figure $2(a)$ and a best-fit ascending straight line on their slope, measuring their intersection. From there, we obtain $x_{c}$, which by its definition is easily converted to $t_{c o n v}$. The fitting of the plateau was sometimes troublesome due to its short duration and the proximity to the initial transient; for this reason, the determination of the transition $R a_{c}$ (or $x_{c}$ ) cannot be made very precisely, which explains the scatter in the data. The experimental points determined by the latter method appear to be more accurate than the ones obtained by the former. However, the spread in data remains within a reasonable tolerance of $\pm 5 \mathrm{~s}$. Nonetheless, the cross-over $R a_{c}$ values increase with increasing $\zeta$ (see figure $3 b$ ). The origin of this behaviour will be explained later in the text.

The gas-depleted region that develops around the bubble is subjected to an upward buoyant force due to its lower density compared to the fluid bulk. In order to estimate the force magnitude, we calculate the volume $V_{b}$ of the buoyant region determined by the spherical segment (of horizontal diameter $2 R+2 \delta$ and height $2 R+\delta$ ) denoted by the dotted line in figure $1(b)$ minus the volume of the bubble. With the bubble radius and buoyant region growing as $R \approx S^{*} \sqrt{2 D c_{s} \zeta t / \rho_{g}}$ and $\delta \approx \sqrt{\pi D t}$, respectively, the 
buoyant volume is given by

$$
V_{b}=\frac{1}{3}(\pi D t)^{3 / 2}\left(24 S^{* 2} \frac{c_{s} \zeta}{\rho_{g}}+9 S^{*} \sqrt{\frac{2 \pi c_{s} \zeta}{\rho_{g}}}+2 \pi\right) \equiv f_{V} t^{3 / 2},
$$

where $f_{V}$ stands for a volumetric buoyant factor. We stress that $f_{V}$ is a dimensional constant that depends on the liquid-gas properties and, most importantly, on the supersaturation $\zeta$.

We then estimate the terminal rising velocity $u_{b}$ of the buoyant region by recognising that the only relevant forces acting upon it are buoyancy $F_{b}$ and the viscous drag $F_{d}$, which for definiteness we estimate from the Stokes' flow. This last assumption is reasonable since the Reynolds number $R e$ is small at all times, as we will see later. The small density difference and 'quasi-static' (extremely slow) growth of $V_{b}$ in the small supersatured regime result in a negligible acceleration, and therefore, negligible added mass force on $V_{b}$. Hence, we define:

$$
F_{b}=c_{s} \zeta \lambda_{c} \rho_{m, 0} g V_{b}, \quad F_{d}=6 \pi u_{b}(R+\delta) \mu,
$$

which once equated lead to

$$
u_{b}=\frac{c_{s} \zeta \lambda_{c} g V_{b}}{6 \pi v(R+\delta)}=\frac{c_{s} \zeta \lambda_{c} g f_{V}}{6 \pi v(R+\delta)} t^{3 / 2} .
$$

Note that, in the end, the final scaling is $u_{b} \propto t$ and that the expression presented above has been written as $u_{b} \propto t^{3 / 2} /(R+\delta)$ for convenience.

The lighter liquid around the bubble will thus rise with the velocity $u_{b}$ which sets up a flow of similar magnitude over the substrate to which the bubble is attached. This will lead to a viscous boundary layer that is described by a Reynolds number

$$
R e=\frac{u_{b}(R+\delta)}{v}=\frac{c_{s} \zeta \lambda_{c} g V_{b}}{6 \pi v^{2}}=\frac{c_{s} \zeta \lambda_{c} g f_{V}}{6 \pi v^{2}} t^{3 / 2} .
$$

Only if the buoyant velocity $u_{b}$ is large enough to overcome the viscous velocity $v /(R+\delta)$ in a boundary layer over a substrate of similar size as the buoyant volume will the liquid start to rise and convective mass transport begin to set in. This implies that $R e>1$, and that the threshold value, i.e. $R e \approx 1$, marks the moment in time $t_{\text {conv }}$ at which convection sets in,

$$
t_{\text {conv }} \approx\left(\frac{6 \pi v^{2}}{c_{s} \zeta \lambda_{c} g f_{V}}\right)^{2 / 3} .
$$

If we consider a pure diffusive growth until the moment of transition, the crossing $R a_{c}$ can be directly calculated by combining (2.3), (3.1) and (3.6),

$$
R a_{c}=\frac{48 \pi \nu S^{* 3}}{D f_{V}}\left(\frac{2 D c_{s} \zeta}{\rho_{g}}\right)^{3 / 2}
$$

an expression which depends only on the properties of the solution and the supersaturation $\zeta$. Note that this occurs because we are performing a quasi-steady-state analysis on a growing bubble with a boundary layer that grows at the same pace. Incidentally, the Reynolds number defined in (3.5) can be easily interpreted as a 

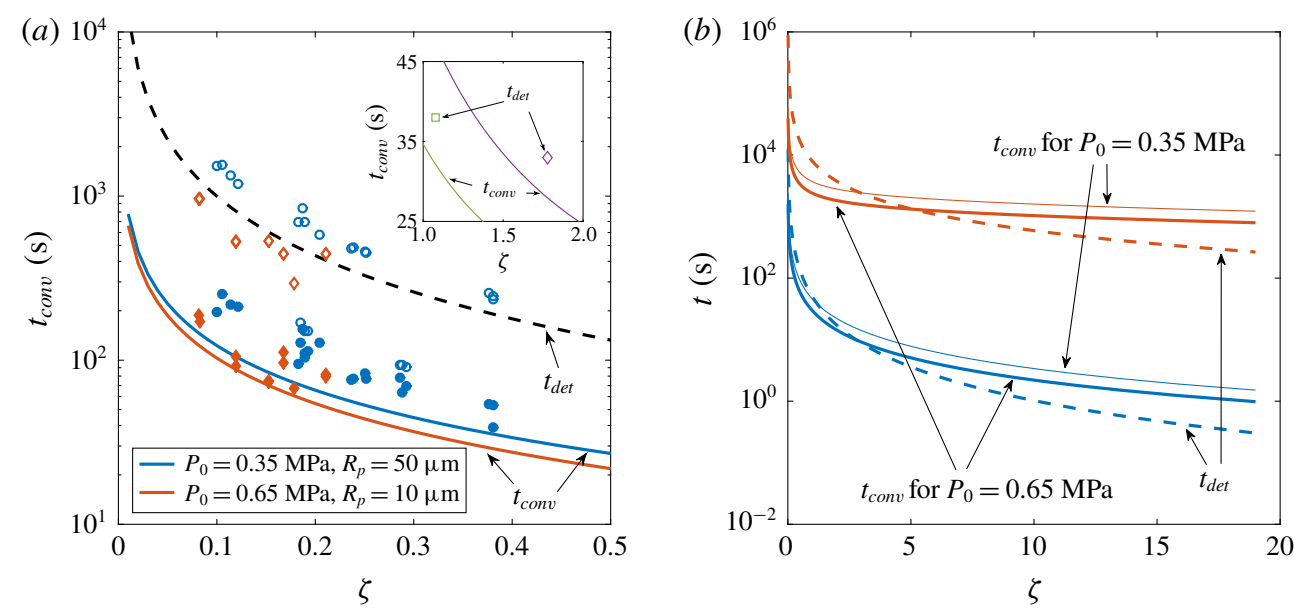

FIgURE 4. (Colour online) (a) Experimental and theoretical convection transition times $t_{\text {conv }}$ (solid symbols and solid lines, respectively) as functions of the supersaturation level $\zeta$ for $P_{0}=0.35 \mathrm{MPa}$ (blue circles) and $P_{0}=0.65 \mathrm{MPa}$ (red diamonds). Open symbols show the experimental detachment times $t_{d e t}$, whereas the dashed line represents the theoretical one for a bubble growing until $R_{\text {det }}$ (2.2) following the growth law (2.3). Inset: detachment times of bubbles in the experiments by Bisperink \& Prins (1994) (diamond) and Jones et al. (1999b) (square). They reported no influence of natural convection. (b) Theoretical cross-over $t_{\text {conv }}$ (solid) and detachment $t_{\text {det }}$ (dashed) times as function of the supersaturation $\zeta$ for $\mathrm{CO}_{2}$ (blue) and $\mathrm{N}_{2}$ (red) bubbles. For thick solid lines, $P_{0}=0.65 \mathrm{MPa}$, and for thin solid ones, $P_{0}=0.35 \mathrm{MPa}$. The detachment curves are calculated considering pure diffusive growth until a radius $R_{d e t}=500 \mu \mathrm{m}(2.2)$, corresponding to a pit radius of $R_{p}=$ $10 \mu \mathrm{m}$.

Grashof number based on the length scale $\left(V_{b} / 6 \pi\right)^{1 / 3}$. By the definition in (3.5), the higher the buoyant velocity $u_{b}$, the earlier and more intensified the onset of convection occurs and the more the mass transfer is enhanced.

In figures $3(b)$ and $4(a)$, we see that the cross-over time prediction agrees well with experimental measurements. In figure $3(b)$, there seems to be a slight dependence on $P_{0}$ which our model (3.7) does not account for. In figure 4(a) we also show, for reference, the experimental detachment times $t_{\text {det }}$ and the theoretical ones if the bubbles would have grown only by diffusion (2.3) to a radius of $500 \mu \mathrm{m}$, i.e. the approximate detachment size (2.2) from a pit of $R_{p}=10 \mu \mathrm{m}$. Furthermore, we include the detachment times of experiments by other authors (Bisperink \& Prins 1994; Jones et al. 1999b) who reported no influence of natural convection during bubble growth. Those times are only slightly larger than our prediction for $t_{c o n v}$, which suggests that in their experiments there was not enough time to observe this phenomenon. In addition, at higher supersaturation values (such as in those studies) the advective flow induced by the expanding bubble surface is no longer negligible and could possibly overwhelm the influence of natural convection.

To analyse the supersaturation range in which convection may become dominant, we compare the Sherwood number $S h$ (3.1) with the Péclet number $P e=2 R \dot{R} / D$, i.e. we compare advective mass transport to the total mass transport:

$$
\frac{P e}{S h}=\frac{c_{s} \zeta}{\rho_{g}}=\zeta k_{H} r_{\mathrm{CO}_{2}} T,
$$




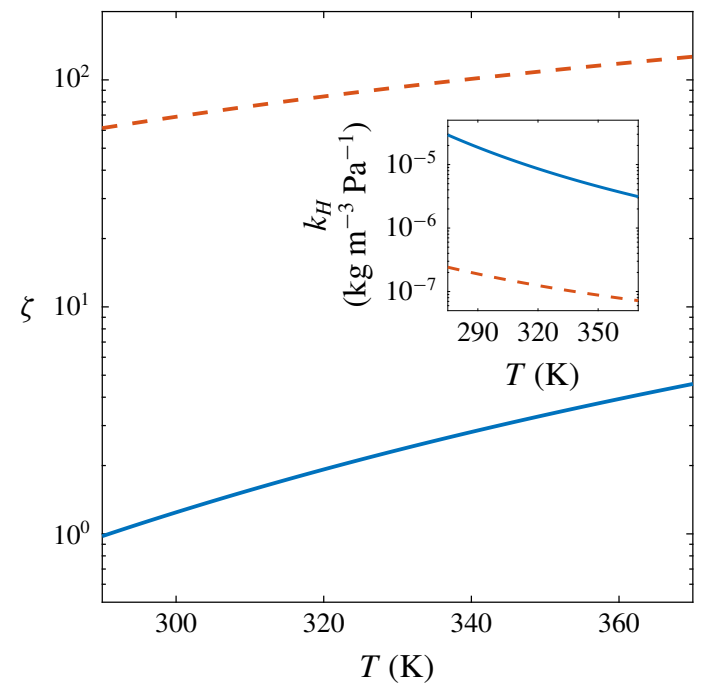

FIgURE 5. (Colour online) Values of $\zeta$ for which $P e / S h=1$ and (inset) Henry's constant $k_{H}$ in water as function of temperature for $\mathrm{CO}_{2}$ (solid blue lines) and $\mathrm{N}_{2}$ (dashed red lines). In both cases, the region $\mathrm{Pe} / \mathrm{Sh}<1$ is below the respective curve.

where we have used both Henry's law and the ideal gas law. Here the specific gas constant for $\mathrm{CO}_{2}$ equals $r_{\mathrm{CO}_{2}}=188.95 \mathrm{~J} \mathrm{~kg} \mathrm{~K}{ }^{-1}$. A necessary condition for natural convection to eventually become dominant is that $\mathrm{Pe} / \mathrm{Sh}<1$, i.e. that advective transport is less relevant than the total mass transfer process including natural convection. Importantly, the threshold only depends on the specific gas, the supersaturation level $\zeta$ and the temperature $T$. In figure 5 we show the temperature dependence of the values of $\zeta$ corresponding to $\mathrm{Pe} / \mathrm{Sh}=1$ and of $k_{H}$. At our experimental temperature $(T \approx 293 \mathrm{~K})$, convection becomes dominant for supersaturation levels below $\zeta=1.08$, including the entire range of our experiments. Therefore, we always observe a transition to density-driven convection during bubble growth. Larger $\zeta$ may then cause advective effects due to the bubble interface expansion and detachment to set in earlier than natural convection. The experiments from Bisperink \& Prins (1994) and Jones et al. (1999b) had supersaturations of 1.78 and 1.07, with corresponding temperatures of 294 and $299 \mathrm{~K}$. For these, $\mathrm{Pe} / \mathrm{Sh}=1.65$ and 0.88 , respectively, which puts them very close to the limit where, given enough time and experimental precision, convection might have been noticeable.

\subsection{Effect of the initial saturation pressure $P_{0}$ on the transition to convection}

As already indicated, the gas concentration in a liquid $c_{0}$ depends on the properties of the gas-liquid couple, the temperature $T$ and the saturation pressure $P_{0}$. At constant $T$, $c_{s}$ is directly proportional to $P_{s}$, and therefore the supersaturation level $\zeta$ can be easily controlled by pressure change. The time evolution of the radius in the diffusive regime is fixed by providing $\zeta$ (Epstein \& Plesset 1950; Enríquez et al. 2014; Moreno Soto et al. 2017). This can be easily realised by noting that in (1.1) and (2.3), the factor $c_{s} \zeta / \rho_{g}=\zeta k_{H} r T$ depends on $\zeta$. The situation is different in the case of buoyancy. Since the buoyant force is directly determined by the density difference $\Delta \rho=\rho_{m, 0} \lambda_{c} c_{s} \zeta=$ $\rho_{m, 0} \lambda_{c} \zeta k_{H} P_{s}$, its effect is expected to depend on both the supersaturation $\zeta$ and the 

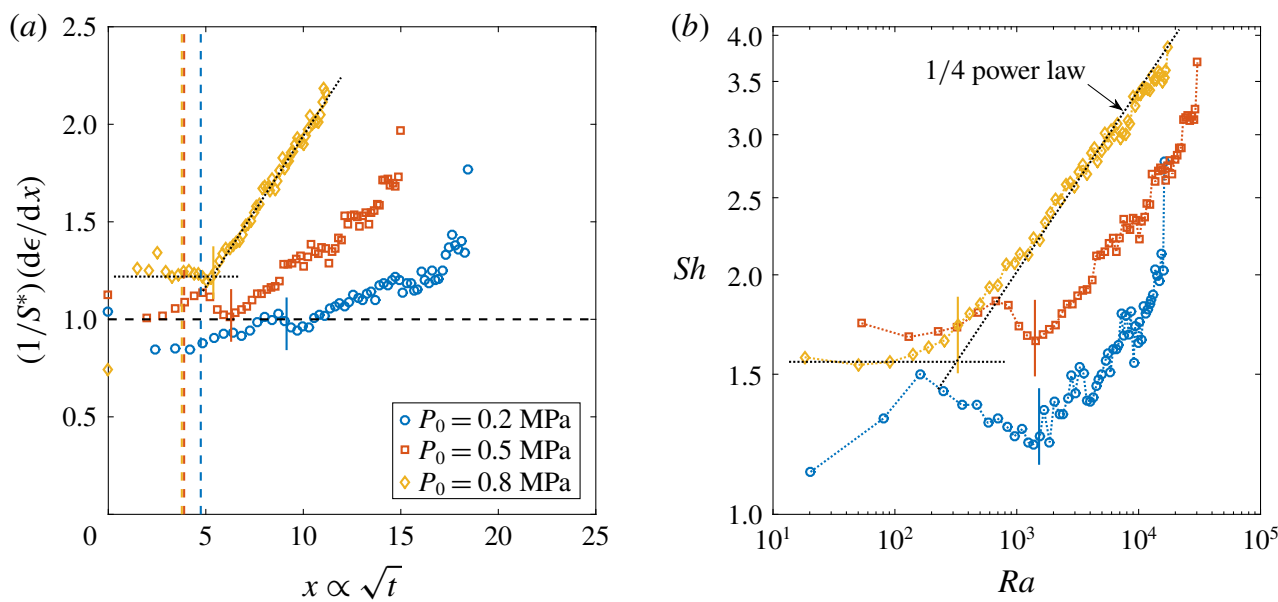

FIgURE 6. (Colour online) (a) Derivative of the dimensionless bubble radius $\epsilon$ against the dimensionless time $x$ for experiments with different $P_{0}$ but same $\zeta \approx 0.15$ and $R_{p}=50 \mu \mathrm{m}$. The corresponding coloured dashed vertical lines stand for the theoretical $t_{\text {conv }}$, whereas the solid lines indicate the experimentally measured one. The guidelines to calculate the latter are indicated as dotted lines for $P_{0}=0.8 \mathrm{MPa}$. The horizontal black dashed line indicates the theoretical behaviour for pure diffusion according to (2.3) (Enríquez et al. 2014). Note that for purely diffusively growing bubbles, the experimental curves would be expected to coincide. (b) $S h$ versus $R a$ for the first bubble after the pressure decrease at different $P_{0}$ and $\zeta=0.15$ in logarithmic scale. The differences present in $(a)$ are directly translated and better visualised in this representation, where for the same $R a, S h$, i.e. the dimensionless mass transfer, increases with higher $P_{0}$. A $1 / 4$ power law has been fitted to one of the experimental curves for comparison with the natural convective behaviour. The transition $R a_{c}$ is calculated as the intersection with the horizontal dotted line which represents a purely diffusive growth. The vertical solid lines in corresponding colour indicate the different $R a_{c}$ for each curve. The same colour palette in $(a)$ applies to $(b)$.

pressure $P_{s}$ (or, alternatively, $P_{0}$ ). This implies that, when by fixing $\zeta$ the zeroth-order diffusive bubble growth is fixed, the time for convection to set in should still depend on the absolute pressure values.

To analyse this phenomenon, we performed experiments at a constant supersaturation level $\zeta=0.15 \pm 0.02$ but initially saturating the solution at a different $P_{0}$. Consequently, the pressure drop to $P_{s}=P_{0} /(\zeta+1)$ is defined from (2.1). Figure 6(a) shows some representative curves of the derivatives of the dimensionless radius $\epsilon$ with respect to the dimensionless time $x$ for three different initial saturation pressures $P_{0}$. The influence of the onset of convection is evident: not only do we observe a shift in the onset time, but even more importantly, the plateau that represents diffusion reaching a higher value for higher $P_{0}$, which indicates an intensified diffusive growth rate associated with larger concentration differences (but still of the same order of $S^{*}$ ). The slope of the convective portion of each curve also increases with $P_{0}$, as can be determined by examining figure $6(b)$. In the latter, the data from figure $6(a)$ are replotted in dimensionless form as $S h$ versus $R a$. Clearly, for the same $R a, S h$ is larger at higher $P_{0}$, indicating a stronger convection and an earlier onset. This intensification of the convective regime with increasing $P_{0}$ originates from the larger concentration change (and consequently, the larger solution density variation) to achieve a constant 

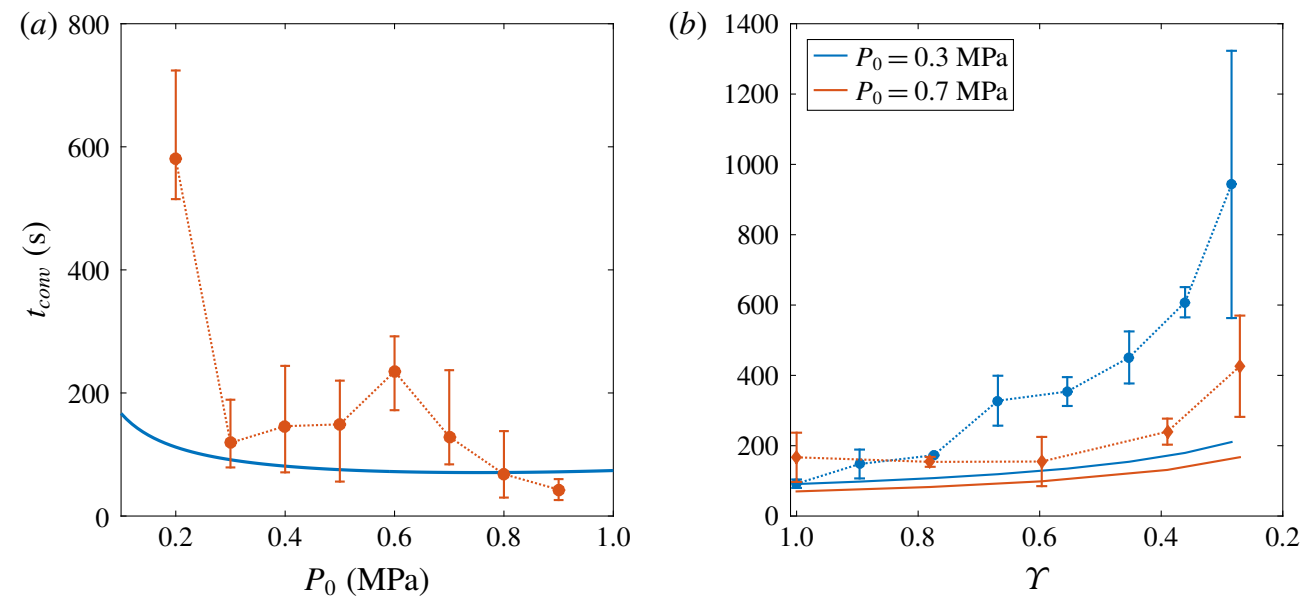

FIGURE 7. (Colour online) (a) Value of $t_{\text {conv }}$ for the different initial saturation pressure $P_{0}$ at $\zeta=0.15$. Experiments are represented by error bars, whereas the theoretical estimation (3.9) is plotted as a solid blue line. Despite the imprecisions in the experimental measurements, especially for lower $P_{0}$, theory agrees qualitatively with experiments, i.e. $t_{c o n v}$ decreases with increasing $P_{0}$. (b) Effect of depletion on the transitional time to convection $\tilde{t}_{\text {conv }}$. Blue circles correspond to $P_{0}=0.3 \mathrm{MPa}$, whereas red diamonds refer to $P_{0}=0.7 \mathrm{MPa}$. For both curves, $\zeta=0.15$. Note that the numbers in the $x$-axis are presented in decreasing order. Even though theory (solid lines) indicates a gradual delay in $\tilde{t}_{c o n v}$, experiments show an intensified effect.

supersaturation level. Thus according to (3.4), the buoyant velocity $u_{b}$ increases with $P_{0}$, which results in a stronger convection and an earlier transition time $t_{c o n v}$. However, as reflected in (3.7), the pressure dependence disappears when defining $R a_{c}$, as the increase in the concentration difference and expansion of the buoyant depleted volume is counteracted by a faster transition time $t_{c o n v}$. The discrepancies in this aspect observed in figure 6(b) still lie within our experimental error.

Turning to the prediction (3.6), we observe that $t_{c o n v}$ is inversely proportional to the gas concentration difference $c_{s} \zeta$. By applying Henry's law, it can be rewritten as $c_{s} \zeta=$ $k_{H} P_{0} \zeta /(\zeta+1)$. Thus, we may reformulate the approximate model (3.6) and obtain:

$$
t_{\text {conv }} \approx\left(\frac{6 \pi v^{2}}{k_{H} P_{0}\left(\frac{\zeta}{\zeta+1}\right) \lambda_{c} g f_{V}}\right)^{2 / 3} .
$$

Note that the volumetric buoyant factor $f_{V}$ (3.2) also depends slightly on $P_{0}$ as the product $c_{s} \zeta / \rho_{g}$ in its definition refers to a difference in concentration $\left(c_{0}-c_{s}\right)$, where $c_{0}$ is directly proportional to $P_{0}$, divided by the gas density, which also depends on this pressure. The experimental results are plotted in figure $7(a)$. Despite the difficulties in measuring a proper $t_{c o n v}$, especially for the lower values of $P_{0}$ in which there is not a clear transition towards convection, theory and experiments follow the same trend and qualitatively agree, i.e. $t_{c o n v}$ decreases with increasing $P_{0}$. 

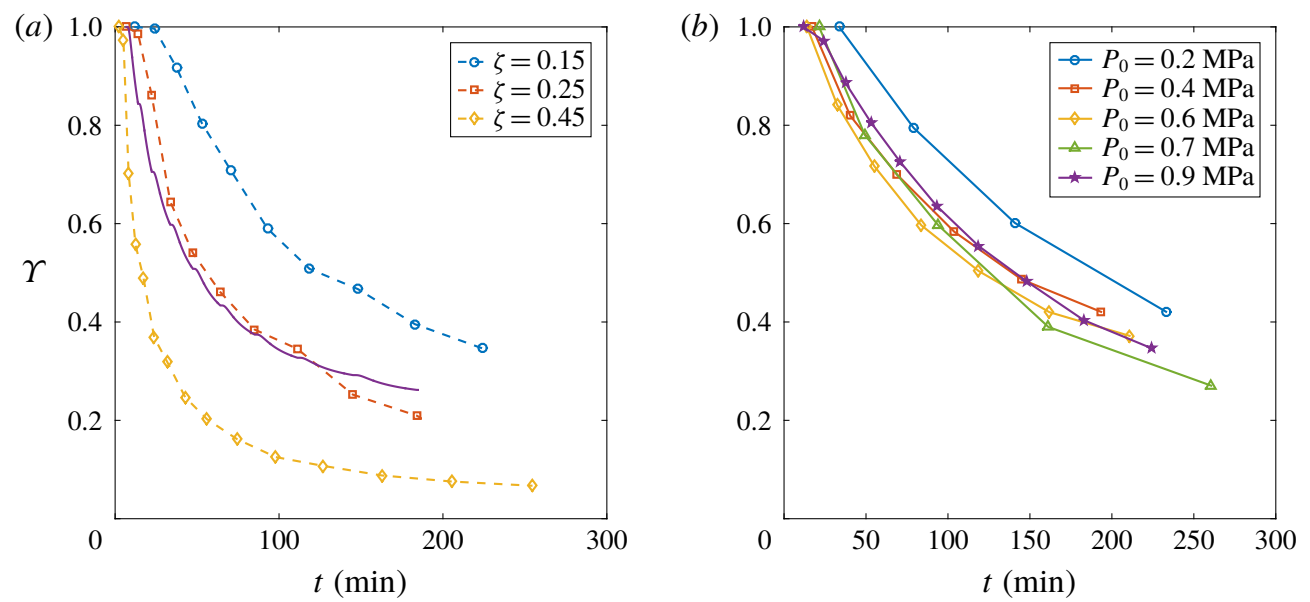

FIgURE 8. (Colour online) (a) Evolution of the depletion number $\Upsilon$ in time with different supersaturation level $\zeta$ and fixed $P_{0}=0.9 \mathrm{MPa}$. An evident faster depletion occurs with larger $\zeta$. The solid line stands for the theoretical behaviour by Moreno Soto et al. (2017) with $\alpha=0.42$ for the case $\zeta=0.25$. (b) Evolution of $\Upsilon$ in time for different starting saturation pressure $P_{0}$ and $\zeta=0.15$. The experimental results make it reasonable to suggest that $P_{0}$, and therefore the intensity of the transition to convection, plays a secondary role in the speed of depletion.

\subsection{Long-term effects on the convective transition during a single bubble succession}

As singles bubbles grow in succession, they will suffer the accumulated depletion which originates from the previous bubbles absorbing the gas from their surroundings. This cumulative depletion remarkably affects subsequent bubbles and significantly delays their growth rate and inhibits the transition to convection (Moreno Soto et al. 2017). We account for the effect of depletion by defining the discrete depletion number $\Upsilon_{n}$ :

$$
\Upsilon_{n}=\frac{\tilde{c}_{n}-c_{s}}{c_{0}-c_{s}},
$$

where $\tilde{c}_{n}$ is the apparent bulk concentration that the $n$th bubble in the succession observes. By definition, the first bubble in the succession, $n=1$, grows in a non-depleted domain and therefore, $\Upsilon_{1}=1$. The subsequent values of $\Upsilon_{n}$ are calculated by collapsing the different curves $S h_{n}$ versus $R a_{n}$ into one universal curve $\widetilde{S h}=f\left(\Upsilon_{n}, \widetilde{R a}\right)$, where $\widetilde{S h}=S h_{n} / \Upsilon_{n}$ and $\widetilde{R a}=R a_{n} \Upsilon_{n}$, following the approach of Moreno Soto et al. (2017), who also calculated a simplified analytical expression for $\Upsilon_{n}$. By obtaining the different values of $\Upsilon_{n}$, figure 8 , different responses can be identified. Firstly, increasing the supersaturation level $\zeta$ at the same starting $P_{0}$ results in a faster depletion, and therefore, $\Upsilon$ decreases faster, as can be seen in figure $8(a)$. Secondly, saturating the solution at different $P_{0}$ does not play a significant role in the evolution of $\Upsilon$, figure $8(b)$. In general, it is true that higher $P_{0}$ results in a slightly slower depletion, except for the case of $P_{0}=0.2 \mathrm{MPa}$, in which depletion is much slower than in the others. We identify this effect as originating from the difficulty in achieving a specific value of $\zeta$, which becomes more challenging at lower $P_{0}$.

Depletion also affects the time at which convection sets in. By assuming that the effective concentration difference $c_{s} \zeta$ which affects the transition to convection is 


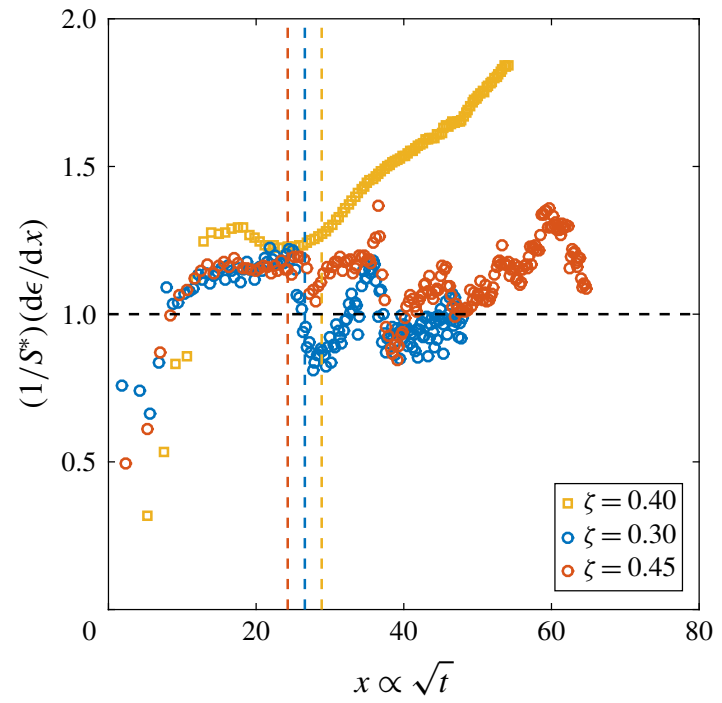

FIGURE 9. (Colour online) Derivative of the dimensionless bubble radius $\epsilon$ versus the dimensionless time $x$ for one $\mathrm{CO}_{2}$ (yellow squares) and two $\mathrm{N}_{2}$ (blue and red circles) bubbles. The vertical dashed lines in corresponding colours indicate the predicted convection cross-over times $x_{\text {conv }}$ for each case (refer to figure $4 b$ ). The horizontal dashed line stands for (2.3).

governed by the apparent bulk concentration $\tilde{c}$, equation (3.6) can be redefined as

$$
\tilde{t}_{c o n v} \approx\left(\frac{6 \pi v^{2}}{\left(\tilde{c}-c_{s}\right) \lambda_{c} g f_{V}}\right)^{2 / 3}=\left(\frac{6 \pi v^{2}}{\Upsilon c_{s} \zeta \lambda_{c} g f_{V}}\right)^{2 / 3}
$$

This equation relates the delay in the transition to convection as depletion intensifies. We plot some experimental results and compare them to this theoretical expression in figure $7(b)$. Despite the toughness in calculating $\tilde{t}_{c o n v}$, which becomes even more difficult as depletion increases and there is no longer a defined transition from plateau to slope in the curves $\mathrm{d} \epsilon / \mathrm{d} x(x)$ nor $\operatorname{Sh}(R a)$ (Moreno Soto et al. 2017), experimental results indicate a significant delay in the transitional time to convection, which is in accordance with (3.11).

\section{The case of $\mathrm{N}_{2}$ bubbles}

$\mathrm{CO}_{2}$ is a gas with a very high solubility in water, indicated by Henry's constant $k_{H}$, whereas $\mathrm{N}_{2}$ has a lower solubility (refer to table 1). The diffusivity $D$ and the expansion coefficient $\lambda_{c}$ in aqueous solutions are however of the same order of magnitude (in absolute terms) for both gases. That $c_{s}$ is approximately one hundred times smaller for $\mathrm{N}_{2}$ leads to a much smaller $c_{s} \zeta$ if the supersaturation $\zeta$ is the same as in the $\mathrm{CO}_{2}$ experiments. Consequently, since the radius grows as $R(t) \propto \sqrt{2 D c_{s} \zeta t / \rho_{g}}, \mathrm{~N}_{2}$ bubbles grow more slowly and $t_{c o n v}$ is expected to be much larger. Figure $4(b)$ compares the theoretical cross-over $t_{c o n v}$ and detachment $t_{d e t}$ times for the two gases, showing the indeed much longer times to achieve convection and detachment for $\mathrm{N}_{2}$. Our estimation of $t_{c o n v}$ remains valid for negative $\lambda_{c}$, where in the final expression (3.6), $\lambda_{c}$ needs to be replaced with its absolute value $\left|\lambda_{c}\right|$. Some experimental $\mathrm{d} \epsilon / \mathrm{d} x$ curves are shown in figure 9 along with a reference curve for 


$\begin{array}{lccc} & t_{\text {conv }}(\mathrm{s}) & R\left(t_{\text {conv }}\right)(\mathrm{mm}) & \delta\left(t_{\text {conv }}\right)(\mathrm{mm}) \\ \mathrm{CO}_{2}, \zeta=0.381 & 60 & 0.300 & 0.610 \\ \mathrm{~N}_{2}, \zeta=0.304 & 3280 & 0.210 & 4.400 \\ \mathrm{~N}_{2}, \zeta=0.457 & 2620 & 0.230 & 4.000\end{array}$

TABLE 2. Comparison of cross-over time $t_{\text {conv }}$, bubble radius at cross-over time $R\left(t_{\text {conv }}\right)$ and boundary layer thickness at cross-over time $\delta\left(t_{\text {conv }}\right)$ for $\mathrm{CO}_{2}$ and $\mathrm{N}_{2}$ bubbles.

$\mathrm{CO}_{2}$. Note that, although the time scales are very different in experiments with each gas, $x$ is similar for $\mathrm{N}_{2}$ and $\mathrm{CO}_{2}$. The early stages look very similar; in fact, the slow growth of $\mathrm{N}_{2}$ bubbles allows for a smoother plateau region, avoiding the slight oscillations of the $\mathrm{CO}_{2}$ curve caused by the pressure controller after the pressure drop. At the predicted $t_{c o n v}$ there is a clear change in behaviour for the $\mathrm{N}_{2}$ bubbles, but very different from that of the $\mathrm{CO}_{2}$ case. In this case, $\lambda_{c}$ is negative and therefore, the stratified density profile is stable: the solution is then denser close to the bubble than in the bulk liquid, i.e. the depleted volume may stay on the substrate and sink. The subsequent convective stream flows in the opposite direction to that in the $\mathrm{CO}_{2}$ case. Still, the transition to natural convection occurs since additional gas is brought from the bulk to the bubble interface. However, the behaviour is totally different from that of $\mathrm{CO}_{2}$ bubbles: now the growth rate oscillates around the solution for purely diffusive growth. Thus, the Riley scaling of the convective flux, i.e. $S h \propto R a^{1 / 4}$, may not apply any longer to this scenario. The different transition times for $\mathrm{CO}_{2}$ and $\mathrm{N}_{2}$ are listed in table 2. As one can see from the data in the table, the cross-over radius $R\left(t_{\text {conv }}\right)$ is of the same order for both gases. However, the cross-over time $t_{\text {conv }}$ and consequently the boundary layer thickness $\delta\left(t_{\text {conv }}\right)$ are much larger for $\mathrm{N}_{2}$.

As we previously mentioned in $\S 2$, it is not uncommon that some bubbles grow on the edges of the silicon substrate during the experiments, which are at least $4 \mathrm{~mm}$ away from the target bubble. For $\mathrm{CO}_{2}$ bubbles this is not a problem, since that distance is much larger than the boundary layer thickness at the time convection sets in, $\delta\left(t_{\text {conv }}\right)$, and hence beyond the range in which they might interact (Enríquez 2015, chap. 5). However for $\mathrm{N}_{2}$, this distance allows for considerable overlap of buoyant regions, which may cause that the mixing due to natural convection brings liquid that is already depleted of gas to the bubble surface. This is consistent with the irregular growth rates and the lack of reproducibility that we observe in all our $\mathrm{N}_{2}$ experiments after $t_{c o n v}$ even though the initial growth is reproducible and similar to $\mathrm{CO}_{2}$ bubbles in dimensionless units (figure 9). In addition, the mixing process itself might be significantly altered in the case of $\mathrm{N}_{2}$ bubbles where, despite the similar magnitude of the buoyant force (in absolute terms), the buoyant depleted volume is much larger than for $\mathrm{CO}_{2}$ bubbles. Finally, and regarding the supersaturation range where diffusion effects should prevail over convection in the case of $\mathrm{N}_{2}$, we can see in figure 5 that the small solubility of this gas in principle sets a very high supersaturation limit to this phenomenon, which again is indicated by the threshold $\mathrm{Pe} / \mathrm{Sh}=1$. The transition to convection is therefore most likely to occur in the majority of applications involving $\mathrm{N}_{2}$ bubbles.

\section{Conclusions}

We have shown that diffusive bubble growth driven by a small gas supersaturation in a liquid solution can lead to natural convection and, thereby, enhance the 
bubble growth rate (figures 2 and $3 a$ ). This transition to natural convection or density-driven convection originates from the change in density experienced by the solution surrounding the bubble as it absorbs the dissolved gas and the liquid becomes depleted. For experiments with $\mathrm{CO}_{2}$, the prediction for the cross-over time $t_{\text {conv }}$ agrees with measurements, despite the difficulty in precisely determining $t_{\text {conv }}$ (figures $3 b$, $4 a$ and $7 a$ ). The analytical prediction of $t_{c o n v}$ suggests that, in principle, density-driven convection around a growing bubble can occur at any value of supersaturation $\zeta$ as long as the detachment radius is large enough (figure 4). However, the balance between the expansive motion of the bubble surface and the convection due to concentration differences establishes a supersaturation threshold for which convection is expected to become relevant (figure 5). The initial saturation pressure $P_{0}$ at which the solution is prepared significantly affects results as well. At constant supersaturation $\zeta$, a higher $P_{0}$ implies a larger pressure drop, and consequently, a larger concentration and density change. As a result, convection sets in at earlier $t_{c o n v}$ and with intensified effects, indicated by the different convective slopes in the derivative analysis (figure $6 a$ ).

Single bubbles growing in a succession are also extremely affected by the transition to convection. The study of the depletion number $\Upsilon$ yields that depletion is highly dependent on the supersaturation level, whereas the initial starting pressure $P_{0}$ has a secondary role and barely influences the results. This sheds some more light in the possible origin of depletion: even though the transition to convection may be relatively important, the major cause of depletion emerges from the evident fact that bubbles absorb gas as they grow (gas that cannot be fully replaced by the diffusive transport through the bulk) and from the way bubbles alter their surroundings while detaching. Besides, depletion causes a significant delay in the transition to convection, which becomes less relevant as subsequent bubbles keep growing and detaching.

For $\mathrm{N}_{2}$ bubbles, there is a clear transition occurring at $t_{\text {conv }}$, consistent with the presence of natural convection, but different from the $\mathrm{CO}_{2}$ case. This originates from the different solubilities and the different way in which the solution density changes with the gas concentration, which is reflected in an opposite sign of $\lambda_{c}$ as that for the $\mathrm{CO}_{2}$ bubbles. The convective plumes flow in the reverse direction and consequently, the bubble evolution during the convective regime and the time needed for it to set in behave differently, as depicted in both tables 1 and 2 and figures $4(b), 5$ and 9. Nevertheless, that we observe such transition with a gas one hundred times less soluble suggests that this can happen for any gas-liquid solution as long as its density changes with the gas concentration.

\section{Acknowledgements}

We want to thank S. Schlautmann for the preparation of the substrates to perform the experiments. This work was supported by the Netherlands Centre for Multiscale Catalytic Energy Conversion (MCEC), an NWO Gravitation programme funded by the Ministry of Education, Culture and Science of the government of the Netherlands.

\section{REFERENCES}

Akin, S. \& Kovscek, A. R. 2002 Heavy-oil solution gas drive: a laboratory study. J. Pet. Sci. Engng 35 (1-2), 33-48.

BARKer, G. S., JefFERSon, B. \& JUdD, S. J. 2002 The control of bubble size in carbonated beverages. Chem. Engng Sci. 57 (4), 565-573. 
Bejan, A. 1993 Heat Transfer, 1st edn. Wiley.

Bisperink, C. G. J. \& PRINS, A. 1994 Bubble growth in carbonated liquids. Colloids Surf. A 85 (2-3), 237-253.

BOLster, D. 2014 The fluid mechanics of dissolution trapping in geologic storage of $\mathrm{CO}_{2}$. J. Fluid Mech. 740, 1-4.

Borkent, B. M., Gekle, S., Prosperetti, A. \& Lohse, D. 2009 Nucleation threshold and deactivation mechanisms of nanoscopic cavitation nuclei. Phys. Fluids 21, 102003.

Dietrich, E., Wildeman, S., Visser, C. W., Hofhuis, K., Kooij, E. S., Zandvliet, H. J. W. \& LOHSE, D. 2016 Role of natural convection in the dissolution of sessile droplets. J. Fluid Mech. 794, 45-67.

EnRíqueZ, O. R. 2015 Growing bubbles and freezing drops: depletion effects and tip singularities. $\mathrm{PhD}$ thesis, Universiteit Twente, The Netherlands.

Enríquez, O. R., Hummelink, C., Bruggert, G.-W., Lohse, D., Prosperetti, A., van der MeER, D. \& Sun, C. 2013 Growing bubbles in a slightly supersaturated liquid solution. Rev. Sci. Instrum. 84, 065111.

Enríquez, O. R., Sun, C., Lohse, D., Prosperetti, A. \& Van Der Meer, D. 2014 The quasi-static growth of $\mathrm{CO}_{2}$ bubbles. J. Fluid Mech. 741 (R1), 1-9.

Epstein, P. S. \& Plesset, M. S. 1950 On the stability of gas bubbles in liquid-gas solutions. J. Chem. Phys. 18 (11), 1505-1509.

Fernández, D., Maurer, P., Martine, M., Coey, J. M. D. \& Möbius, M. E. 2014 Bubble formation at a gas-evolving microelectrode. Langmuir 30 (43), 13065-13074.

Fritz, W. 1935 Berechnung des Maximal Volume von Dampfblasen. Phys. Z. 36, 379-388.

Gebhart, B. \& PeRA, L. 1971 The nature of vertical natural convection flows resulting from the combined buoyancy effects of thermal and mass diffusion. Intl J. Heat Mass Transfer 14 (12), 2025-2050.

Greenwood, N. N. \& Earnshaw, A. 1997 Chemistry of the Elements, 2nd edn. Elsevier Butterworth-Heinemann.

Jansen, H., De Boer, M., Legtenberg, R. \& Elwenspoek, M. 1995 The black silicon method: a universal method for determining the parameter setting of a fluorine-based reactive ion etcher in deep silicon trench etching with profile control. J. Micromech. Microengng 5 (2), 115-120.

Jones, S. F., Evans, G. M. \& GAlvin, K. P. $1999 a$ Bubble nucleation from gas cavities - a review. Adv. Colloid Interface Sci. 80 (1), 27-50.

Jones, S. F., Evans, G. M. \& Galvin, K. P. $1999 b$ The cycle of bubble production from a gas cavity in a supersaturated solution. Adv. Colloid Interface Sci. 80 (1), 51-84.

LeEnheER, A. J. \& ATwATER, H. A. 2010 Water-splitting photoelectrolysis reaction rate via microscopic imaging of evolved oxygen bubbles. J. Electrochem. Soc. 157 (9), B1290-B1294.

Li, J., Chen, H., Zhou, W., Wu, B., Stoyanov, S. D. \& Pelan, E. G. 2014 Growth of bubbles on a solid surface in response to a pressure reduction. Langmuir 30 (15), 4223-4228.

van der Linde, P., Peñas-López, P., Moreno Soto, Á., VAn Der Meer, D., Lohse, D., GARDENIERS, H. \& FERnÁndez Rivas, D. 2018 Gas bubble evolution on microstructured silicon substrates. Energy Environ. Sci. 11 (12), 3452-3462.

Lohse, D. \& Zhang, X. 2015 Surface nanobubbles and nanodroplets. Rev. Mod. Phys. 87 (3), 981-1035.

Moreno Soto, Á., Prosperetti, A., Lohse, D. \& van Der Meer, D. 2017 Gas depletion through single gas bubble diffusive growth and its effect on subsequent bubbles. J. Fluid Mech. 831, 474-490.

Munasinghe, P. C. \& Khanal, S. K. 2010 Biomass-derived syngas fermentation into biofuels: opportunities and challenges. Bioresour. Technol. 101 (13), 5013-5022.

Neufeld, J. A., Hesse, M. A., Riaz, A., Hallworth, M. A., Tchelepi, H. A. \& Huppert, H. E. 2010 Convective dissolution of carbon dioxide in saline aquifers. Geophys. Res. Lett. 37, L22404.

Peñas-López, P., Moreno Soto, Á., Parrales, M. A., van der Meer, D., Lohse, D. \& RodrígueZ-RodrígueZ, J. 2017 The history effect in bubble growth and dissolution. Part 2. 
Experiments and simulations of a spherical bubble attached to a horizontal flat plate. J. Fluid Mech. 820, 479-510.

PierantozzI, R. 2007 Carbon dioxide. In Kirk-Othmer Encyclopedia of Chemical Technology, 5th edn. (ed. Kirk-Othmer), vol. 4, pp. 803-822. Wiley.

Pooladi-Darvish, M. \& Firoozabadi, A. 1999 Solution-gas drive in heavy oil reservoirs. J. Can. Pet. Technol. 38 (4), 54-61.

Popov, Y. O. 2005 Evaporative deposition patterns: spatial dimensions of the deposit. Phys. Rev. E 71, 036313.

Potter, J. M. \& Riley, N. 1980 Free convection from a heated sphere at large Grashof number. J. Fluid Mech. 100 (4), 769-783.

SANDER, R. 2015 Compilation of Henry's law constants (version 4.0) for water as solvent. Atmos. Chem. Phys. 15 (8), 4399-4981.

Shahidzadeh-Bonn, N., Rafaï, S., Azouni, A. \& Bonn, D. 2006 Evaporating droplets. J. Fluid Mech. 549, 307-313.

Somorjai, G. A. \& LI, Y. 2010 Introduction to Surface Chemistry and Catalysis, 2nd edn. Wiley.

SPURGEON, J. M. \& LEWIS, N. S. 2011 Proton exchange membrane electrolysis sustained by water vapor. Energy Environ. Sci. 4 (8), 2993-2998.

Stauber, J. M., Wilson, S. K., Duffy, B. R. \& Sefiane, K. 2014 On the lifetimes of evaporating droplets. J. Fluid Mech. 744 (R2), 1-12.

Tsai, P. A., Riesing, K. \& Stone, H. A. 2013 Density-driven convection enhanced by an inclined boundary: implications for geological $\mathrm{CO}_{2}$ storage. Phys. Rev. E 87, 011003(R).

Watanabe, H. \& IizuKa, K. 1985 The influence of dissolved gases on the density of water. Metrologia 21 (1), 19-26.

Wilke, C. R. \& Chang, P. 1955 Correlation of diffusion coefficients in dilute solutions. AIChE J. 1 (2), 264-270. 